\title{
A coupled lattice Boltzmann and particle level set method for free-surface flows
}

\author{
Yang Yu, Li Chen, Jianhua Lu, Guoxiang Hou* \\ School of Naval Architecture and Ocean Engineering, Huazhong University of Science and Technology, \\ Wuhan, 430074, China \\ *Corresponding author, e-mail: houguoxiang@163.com
}

Received 1 Apr 2013

Accepted 3 Mar 2014

\begin{abstract}
Free-surface flows are very important in many fields. Compared with volume of fluid methods, level set methods are more accurate. In this paper, we develop a coupled lattice Boltzmann and particle level set method to simulate free-surface flows. The effectiveness of this model is verified by simulating the coalescence of two stationary droplets, evolution of an initially circular fluid patch, and the classical breaking-dam case. Results show that this model can be applied to real world simulations with satisfactory accuracy.
\end{abstract}

KEYWORDS: interface capturing, single-phase free-surface, surface tension, breaking dam

\section{INTRODUCTION}

Numerical simulation of free-surface flows is challenging. To solve this problem, various methods, such as the finite difference method, the finite volume method, the smoothed particle hydrodynamics method and the lattice Boltzmann method (LBM), have been proposed. Among them, the newly developed LBM shows great potential for its efficiency, natural parallelism, and simple implementation of boundary conditions.

With the development of the LBM, several multiphase models ${ }^{1-3}$ have been proposed. However, these multiphase models can only deal with flows with small density and viscosity ratios, such as free-surface flows. As to a free-surface flow, the gas phase has a negligible effect on the liquid phase, so it can be ignored during the calculation. For model free-surface flows, some single-phase free-surface models have been developed. Ginzburg ${ }^{4}$ proposed a single-phase free-surface model on the basis of the generalized lattice Boltzmann method. This model requires a complicated recolouring step during the movement of the interface, which significantly lowers the efficiency of the method. Another single-phase free-surface model was proposed in Refs. 5, 6. In this model, the movement of the interface is achieved through a mass exchange between interface and liquid cells during the streaming step of the LBM, and it has a simple but efficient redistribution process. The unknown distribution functions (DFs) from gas cells are reconstructed through a free-surface boundary condition.
Hence this model can simulate free-surface flows with a high computational efficiency and relatively low memory. Since both of the models use the volumetric fraction in the volume of fluid (VOF) method, they can be seen as VOF-type methods.

On the other hand, as an alternative choice of VOF method to capture moving interface, the level set method (LSM) was proposed ${ }^{7}$. The movement of the interface is accomplished through the evolution of a distance function $\phi$. Since the distance function is continuous across the interface, high accuracy can be achieved near the interface. Hence LSM can give a smoother interface than VOF. The major weakness of LSM is the loss of mass during the interface evolution. To handle this problem, a reinitialization process $^{8}$ and a velocity extension process ${ }^{9}$ were developed. However, even with these techniques, the loss of mass is still the main problem. Since mass conservation in a VOF method can be obtained, a coupled level set and volume-of-fluid method (CLSVOF) was proposed ${ }^{10}$. Another way to eliminate the loss of mass is the particle level set method (PLSM) ${ }^{11,12}$. In this method, a number of massless particles are set near both sides of the interface and move with the flow field. Mass conservation is assured by correcting the interface through these particles. Compared with CLSVOF, PLSM is easier for implementation.

In this study, to obtain a more accurate and easierto-implement method to simulate free-surface flows, we combine LBM with PLSM. The effectiveness of this model will be verified with the coalescence of two stationary droplets, the evolution of an initially 
circular fluid patch, and the breaking dam case.

\section{THE LATTICE BOLTZMANN METHOD}

Unlike the conventional macroscopic methods, LBM derives from the microscopic Boltzmann equation and is often referred to as a mesoscopic method. Through a BGK approximation and a finite difference discretization, the Boltzmann equation leads to the following evolution equation for $\mathrm{LBM}^{13}$ :

$$
\begin{aligned}
f_{i}\left(\mathbf{x}+\mathbf{e}_{i} \Delta t, t+\Delta t\right) & -f_{i}(\mathbf{x}, t)= \\
& -\frac{1}{\tau}\left[f_{i}(\mathbf{x}, t)-f_{i}^{(\mathrm{eq})}(\mathbf{x}, t)\right]
\end{aligned}
$$

where $f_{i}(\mathbf{x}, t)$ is the distribution function (DF) at position $\mathbf{x}$ and time $t$ for particles with velocity $\mathbf{e}_{i}$ along the $i$ th direction of the lattice, $f_{i}^{(\text {eq })}$ is the equilibrium distribution function, $\tau$ is the dimensionless relaxation time, and $\Delta t$ is the time step.

Since only two-dimensional cases are considered in this study, the general D2Q9 model is used. For the standard D2Q9 model, the equilibrium distribution function (EDF) can be expressed as ${ }^{14}$

$$
E_{i}(\rho, \mathbf{u})=\omega_{i} \rho\left[1+\frac{\mathbf{e}_{i} \cdot \mathbf{u}}{c_{\mathrm{s}}^{2}}+\frac{\left(\mathbf{e}_{i} \cdot \mathbf{u}\right)^{2}}{2 c_{\mathrm{s}}^{4}}-\frac{\mathbf{u}^{2}}{2 c_{\mathrm{s}}^{2}}\right]
$$

where $\rho$ is the fluid density, $\mathbf{u}$ is the fluid velocity, $\omega_{i}$ is the weight parameter with $\omega_{i}=4 / 9(i=0)$, $1 / 9(i=1,2,3,4), 1 / 36(i=5,6,7,8)$, and the particle velocity $\mathbf{e}_{i}$ is defined as

$$
\left\{\begin{array}{lll}
(0,0), & i=0, \\
\left(\cos \left[\frac{(i-1) \pi}{2}\right], \sin \left[\frac{(i-1) \pi}{2}\right]\right) c, & i=1-4, \\
\sqrt{2}\left(\cos \left[\frac{(2 i-1) \pi}{4}\right], \sin \left[\frac{(2 i-1) \pi}{4}\right]\right) c, & i=5-8
\end{array}\right.
$$

where $c=\Delta x / \Delta t$ is the lattice speed, $\Delta x$ is the grid space, and $c_{\mathrm{s}}=c / \sqrt{3}$ is the speed of sound. Macroscopic variables, $\rho$ and $\mathbf{u}$, can be obtained by the moments of DF

$$
\rho=\sum_{i} f_{i}, \quad \rho \mathbf{u}=\sum_{i} \mathbf{e}_{i} f_{i} .
$$

In addition, by setting the viscosity $\nu$

$$
\nu=(\tau-1 / 2) c_{\mathrm{s}}^{2} \Delta t .
$$

LBM reduces to the Navier-Stokes equation up to second order through a Chapman-Enskog expansion.

Body forces, such as gravity, can be modelled by adding an additional term to $(1)^{15}$ :

$$
\begin{aligned}
& f_{i}\left(\mathbf{x}+\mathbf{e}_{i} \Delta t, t+\Delta t\right)-f_{i}(\mathbf{x}, t)= \\
& -\frac{1}{\tau}\left[f_{i}(\mathbf{x}, t)-f_{i}^{(\mathrm{eq})}(\mathbf{x}, t)\right]+F_{i} \Delta t
\end{aligned}
$$

where $F_{i}$ is

$$
F_{i}=\left(1-\frac{1}{2 \tau}\right) \omega_{i}\left[\frac{\mathbf{e}_{i}-\mathbf{v}}{c_{\mathrm{s}}^{2}}+\frac{\mathbf{e}_{i} \cdot \mathbf{v}}{c_{\mathrm{s}}^{4}} \mathbf{e}_{i}\right] \cdot \mathbf{F}
$$

with

$$
\rho=\sum_{i} f_{i}, \quad \rho \mathbf{u}=\sum_{i} \mathbf{e}_{i} f_{i}+\frac{1}{2} \mathbf{F} \Delta t
$$

where $\mathbf{F}$ is the body force.

For the LBM we divide the evolution equation (6) into collision and stream steps, respectively:

$$
\begin{array}{r}
f_{i}^{\prime}(\mathbf{x}, t)=f_{i}(\mathbf{x}, t)-\frac{f_{i}(\mathbf{x}, t)-f_{i}^{(\mathrm{eq})}(\mathbf{x}, t)}{\tau}+\Delta t F_{i}, \\
f_{i}\left(\mathbf{x}+\mathbf{e}_{i} \Delta t, t+\Delta t\right)=f_{i}^{\prime}(\mathbf{x}, t) .
\end{array}
$$

Through the collision and stream steps, LBM can simulate the evolution of a fluid. Further details can be found in Refs. 14, 15 .

\section{THE PARTICLE LEVEL SET METHOD}

In the level set method, a distance function $\phi$ is defined as ${ }^{16}$

$$
\phi(\mathbf{x})= \begin{cases}0, & \forall \mathbf{x} \in \Gamma, \\ -d(\mathbf{x}), & \forall \mathbf{x} \in \Omega, \\ d(\mathbf{x}), & \forall \mathbf{x} \notin \Omega\end{cases}
$$

where $d(\mathbf{x})=\min \left(\left|\mathbf{x}-\mathbf{x}^{\prime}\right|\right)$ for all $\mathbf{x}^{\prime} \in \Gamma$, and $\Gamma$ represents the interface, and $\Omega$ refers to the space inside $\Gamma$. The movement of the interface is accomplished through the level set equation ${ }^{7}$

$$
\frac{\partial \phi}{\partial t}+\mathbf{u} \cdot \nabla \phi=0
$$

where $\mathbf{u}$ is the velocity of the flow field. In this way, we can simulate the evolution of a two-phase flow.

Since the level set function will not satisfy (10) after several time steps, a reinitialization step should be done at every time step. However, the loss of mass occurring in the level set method can fundamentally affect the accuracy of the interface evolution. To address this issue, the particle level set method was proposed.

In the particle level set method ${ }^{11,12}$, two sets of massless particles, plus and minus particles, are placed near the interface (the place where $\phi=0$ ). Once there is a minus (plus) particle leaking out to the plus (minus) region of the flow filed, a correction step is done to reduce the error of the evolution equation (11). As a result, the loss of mass can be alleviated for the most part.

The particle correction procedures in the particle level set method are summarized as follows: 
(i) Initialization of particles. Particles are placed only within the distance of $3 \max (\Delta x, \Delta y)$ from the interface. We set 16 particles (2D) in a cell. The radius of a particle $r_{\mathrm{p}}$ is given by

$$
r_{\mathrm{p}}= \begin{cases}r_{\max }, & s_{\mathrm{p}} \phi\left(\mathbf{x}_{\mathrm{p}}\right)>r_{\max }, \\ s_{\mathrm{p}} \phi\left(\mathbf{x}_{\mathrm{p}}\right), & r_{\min } \leqslant s_{\mathrm{p}} \phi\left(\mathbf{x}_{\mathrm{p}}\right) \leqslant r_{\max }, \\ r_{\min }, & s_{\mathrm{p}} \phi\left(\mathbf{x}_{\mathrm{p}}\right)<r_{\min }\end{cases}
$$

where $r_{\min }=0.1 \min (\Delta x, \Delta y), r_{\max }=$ $0.5 \min (\Delta x, \Delta y)$, and $s_{\mathrm{p}}$ is the sign of a particle (for $\phi\left(\mathbf{x}_{\mathrm{p}}\right)>0, s_{\mathrm{p}}=+1$; for $\phi\left(\mathbf{x}_{\mathrm{p}}\right)<0$, $\left.s_{\mathrm{p}}=-1\right)$. When this step is done, particles are attracted to the correct side of the interface.

(ii) Identification of error. When the level set function is solved, we need to check whether the loss of mass occurs. If the level set function $\phi$ in the current particle position has a different sign, i.e., $\phi\left(\mathbf{x}_{\mathrm{p}}\right) \cdot s_{\mathrm{p}}<0$, the loss of mass occurs. Then we proceed to the next step.

(iii) Quantification of error. In this step, for a particle moving across the interface with radius $r_{\mathrm{p}}$ and position $x_{\mathrm{p}}$, we define the particle boundary (a circle in 2D) using a level set function

$$
\phi_{\mathrm{p}}(\mathbf{x})=s_{\mathrm{p}}\left(r_{\mathrm{p}}-\left|\mathbf{x}-\mathbf{x}_{\mathrm{p}}\right|\right) .
$$

Since these local values of $\phi_{\mathrm{p}}$ are from the prediction of the level set function on cell corners, possible errors can be identified from the deviation of $\phi$ from $\phi_{\mathrm{p}}$.

(iv) Reduction of error. Escaped plus (minus) particles are used to correct the $\phi>0(\phi<0)$ region. For all the escaped plus particles, we calculate the $\phi_{\mathrm{p}}$ value on cell corners containing the escaped particles using (12), compare each $\phi_{\mathrm{p}}$ with the local $\phi$, and set $\phi_{+}$as the maximum value of these two values. For a set of escaped plus particles $E^{+}$, we initialize $\phi^{+}$with $\phi$ and set

$$
\phi^{+}=\max _{\forall p \in E^{+}}\left(\phi_{\mathrm{p}}, \phi^{+}\right) .
$$

In the same manner, for a set of escaped minus particles $E^{-}$, we also initialize $\phi^{-}$with $\phi$ and set

$$
\phi^{-}=\min _{\forall p \in E^{-}}\left(\phi_{\mathrm{p}}, \phi^{-}\right) .
$$

Then, for each grid point (cell corners), we choose the minimum absolute value as the final correction for $\phi$

$$
\phi= \begin{cases}\phi^{+}, & \left|\phi^{+}\right| \leqslant\left|\phi^{-}\right|, \\ \phi^{-}, & \left|\phi^{+}\right| \geqslant\left|\phi^{-}\right| .\end{cases}
$$

Further details of this method can be found in Refs. 11,12 .

\section{THE COUPLED LATTICE BOLTZMANN AND PARTICLE LEVEL SET METHOD FOR FREE-SURFACE FLOWS}

In this study, to model free-surface flows, three kinds of cells are defined: liquid cells, gas cells, and interface cells. Gas cells are not calculated.

When implementing this single-phase freesurface model, because only one-phase (liquid phase) is simulated, we need to reconstruct the unknown DFs at interface cells from the direction of the gas cells. To achieve this goal, we need to balance mass and momentum between two sides of the free-surface. Following the idea in Ref. 6, we adopt the following free-surface boundary condition:

$$
\begin{aligned}
f_{I}(\mathbf{x}, t+\Delta t)= & f_{i}^{(\mathrm{eq})}\left(\rho_{f}, \mathbf{u}_{\mathrm{B}}\right) \\
& +f_{I}^{(\mathrm{eq})}\left(\rho_{f}, \mathbf{u}_{\mathrm{B}}\right)-f_{i}(\mathbf{x}, t)
\end{aligned}
$$

where $I$ denotes the inverse direction of $i, \rho_{f}$ is the reference density of the liquid and $\mathbf{u}_{\mathrm{B}}$ is taken as the velocity of the interface cell for simplicity. From (16), the liquid pressure and gas pressure at the freesurface is the same but with opposite direction, which means the boundary conditions on the free-surface are fulfilled $^{5}$.

Another issue when combining LBM and PLSM is the extrapolation of velocity. The movement of the interface through LSM requires velocities on both sides of the interface. But for single-phase freesurface flows, velocities in the gas phase are unknown. This problem is solved by using the fast velocity extension method proposed by Adalsteinsson ${ }^{9}$. This extension method has the following form:

$$
\nabla F_{\text {ext }} \cdot \nabla \phi=0
$$

where $F_{\text {ext }}$ means the velocity field to be extended. Eq. (17) can be solved through a fast marching method $^{8,9}$. Eq. (17) not only matches the given velocity on the front (the moving interface), but also preserves the signed distance function (level set function $)^{9}$. Hence (17) is very suitable for the velocity extension in the gas phase.

The level set equation (Eq. (11)) is usually solved by high-order accurate numerical methods such as the TVD Runge-Kutta and HJ-WENO schemes. But with PLSM, a fast first-order semi-Lagrangian advection method is accurate enough and more efficient ${ }^{12}$. For a grid point $\mathbf{x}_{i, j}=(i \Delta x, j \Delta y)$, this semi-Lagrangian scheme takes the form

$$
\begin{gathered}
\phi_{i, j}^{n+1}=\alpha \beta \phi_{r+1, s+1}^{n}+(1-\alpha) \beta \phi_{r, s+1}^{n}+ \\
\alpha(1-\beta) \phi_{r+1, s}^{n}+(1-\alpha)(1-\beta) \phi_{r, s}^{n}
\end{gathered}
$$


with

$$
\begin{array}{ll}
r=i-\left\lceil u_{i, j} \frac{\Delta t}{\Delta x}\right\rceil, & \alpha=\frac{(i-r) \Delta x-u_{i, j} \Delta t}{\Delta x} \\
s=j-\left\lceil v_{i, j} \frac{\Delta t}{\Delta y}\right\rceil, & \beta=\frac{(j-s) \Delta y-v_{i, j} \Delta t}{\Delta y}
\end{array}
$$

where $\mathbf{u}\left(\mathbf{x}_{i, j}\right)=\left(u_{i, j}, v_{i, j}\right),\lceil x\rceil$ means the smallest integer value that is not less than $x$, and $\Delta y=\Delta x$ in current work. According to the Lax-Richtmyer theorem, (18) is unconditionally stable ${ }^{12}$.

Particles are evolved according to Ref. 12

$$
\frac{d \mathbf{x}_{\mathrm{p}}}{\mathrm{d} t}=\mathbf{u}\left(\mathbf{x}_{\mathrm{p}}\right)
$$

where $\mathbf{x}_{\mathrm{p}}$ is the position of a particle, and $\mathbf{u}\left(\mathbf{x}_{\mathrm{p}}\right)$ is the velocity of the particle. Velocities are interpolated by a bilinear interpolation method (2D case). This equation is solved by a third-order TVD Runge-Kutta method.

In this study, the PLSM solver uses the same uniform grid and time step as the LBM solver does. As a result, no conversions for macro variables and DFs are needed unlike in Kwak's work (multi-resolution for PLSM) ${ }^{17}$.

One step for the coupled LBM and PLSM (LBPLSM) is as follows:

Step 1: LBM solver implements collision and stream step (9) and reconstructs unknown distribution functions for interface cells (16).

Step 2: Macroscopic variables, densities $\rho(\mathbf{x}, t)$ and velocities $\mathbf{u}(\mathbf{x}, t)$ are calculated from (8).

Step 3: Velocities of the gas phase $\mathbf{u}_{\mathrm{ext}}(\mathbf{x}, t)$ are extrapolated using (17).

Step 4: Using the extrapolated velocity field, the PLSM solver computes the distance function $\phi$ by solving the level set equation (Eq. (11), (18)). Particles are also advected in this step (Eq. (19)).

Step 5: PLSM solver corrects the interface according to the positions of particles (Eq. (12)-(15)).

\section{RESULTS AND DISCUSSION}

In this section, the coupled LBM and PLSM singlephase free-surface model is implemented to simulate the coalescence of two stationary droplets, the evolution of an initially circular fluid patch, and the classical breaking dam case. The effectiveness of the techniques presented in the previous sections will be evaluated.

\section{Coalescence of two stationary droplets driven by surface tension}

We now look at the coalescence process of two adjacent circular droplets (with radius $R$ ) under the

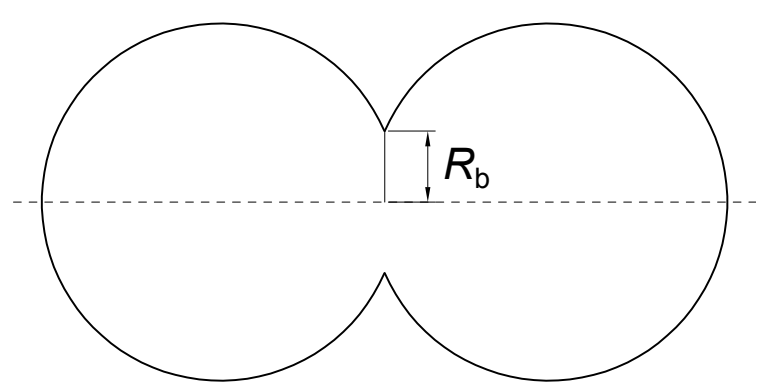

Fig. 1 The bridge radius $R_{\mathrm{b}}$ of the coalescence problem.

influence of surface tension. According to Eggers ${ }^{18}$, the bridge radius (defined as $R_{\mathrm{b}}$ in Fig. 1) of the coalescence problem obeys a scaling law, $R_{\mathrm{b}} \propto$ $(\sigma R / \rho)^{1 / 4} t^{1 / 2}$. Hence for constant surface tension coefficient $\sigma$, we have $R_{\mathrm{b}} \propto t^{1 / 2}$. Wu and $\mathrm{Ho}^{19}$ prove this scaling law through an experiment using a highspeed imaging system. It should be noted that this scaling law only takes effect when the bridge radius is increasing.

To implement the case, we need to describe the surface tension model in the level set method. The momentum equation for incompressible flows with surface tension can be expressed as ${ }^{10}$

$$
\begin{aligned}
\rho\left[\frac{\partial \mathbf{u}}{\partial t}+\right. & \left.\frac{\partial(\mathbf{u u})}{\partial t}\right]=-\nabla p \\
& +\rho \nu \nabla \cdot\left[\nabla \mathbf{u}+(\nabla \mathbf{u})^{\mathrm{T}}\right]+\sigma \kappa \nabla H
\end{aligned}
$$

where $\sigma$ is the surface tension coefficient, $\kappa$ is the mean curvature of the interface, $H$ is the Heaviside function, and $\nu$ is the shear viscosity. $\kappa$ is defined as

$$
\kappa=\nabla \cdot\left(\frac{\nabla \phi}{|\nabla \phi|}\right)
$$

and $H$ is

$$
H= \begin{cases}0, & \phi<-\varepsilon, \\ \frac{1}{2}\left[1+\frac{\phi}{\varepsilon}+\frac{1}{\pi} \sin (\pi \phi / \varepsilon)\right], & |\phi| \leqslant \varepsilon, \\ 1, & \phi>\varepsilon\end{cases}
$$

where $\varepsilon$ is the thickness of the interface.

Since surface tension is treated as a discrete body force, it can be modelled by (6)-(8).

We adopt LB-PLSM and the surface tension model discussed above to simulate this coalescence process. At the beginning of the case, two circular droplets are placed at rest close to each other. Due to the influence of surface tension, these two droplets coalesce into a single droplet. Physical parameters used here are liquid density $\rho=1000 \mathrm{~kg} / \mathrm{m}^{3}$, viscosity 


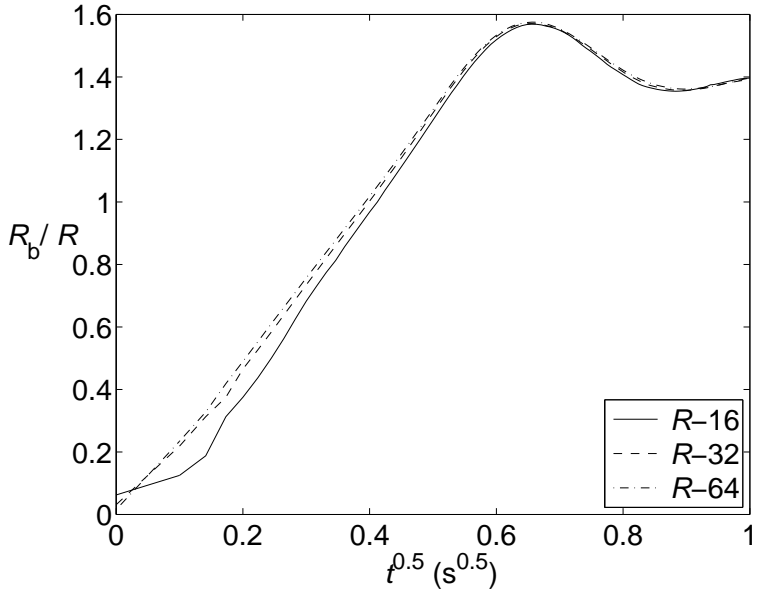

Fig. 2 The bridge radius versus the square root of time for the droplets coalescence problem with three different grid resolutions for $R$.

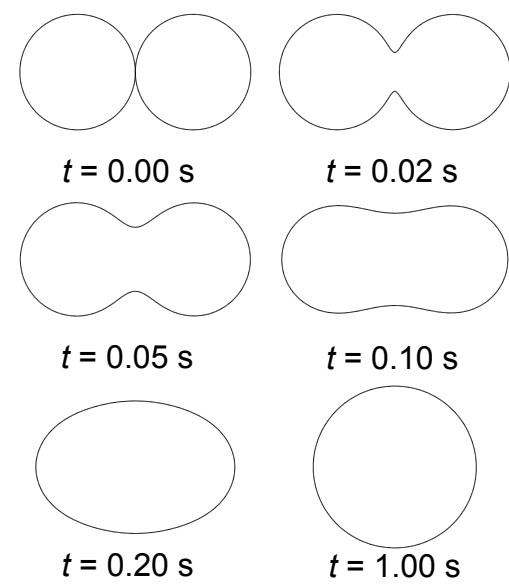

Fig. 3 The evolution process of the coalescence problem with $\sigma=7 \times 10^{-4} \mathrm{~kg} / \mathrm{s}^{2}$ and a grid size of 64 for $R$.

$\nu=1 \times 10^{-5} \mathrm{~m}^{2} / \mathrm{s}$, surface tension coefficient $\sigma=$ $7 \times 10^{-4} \mathrm{~kg} / \mathrm{s}^{2}$, radii of droplets $R=0.002 \mathrm{~m}$. The simulations are implemented on a uniform grid with space step $\Delta x=1$ and time step $\Delta t=1$. Three different grid resolutions $(16,32,64)$ for $R$ with corresponding dimensionless relaxation time coefficient $\tau(0.74,0.98,1.46)$ are adopted.

From Fig. 2, we can see that, with the lowest grid resolution, the bridge radius does not follow the scaling law until $t^{0.5}>0.3$. However, with the refinement of grid resolution, the bridge radius obeys the scaling law even from the start of the simulation, which suggests the convergence of the current model. The evolution process of the coalescence problem with a grid size of 64 for $R$ is displayed in Fig. 3 .

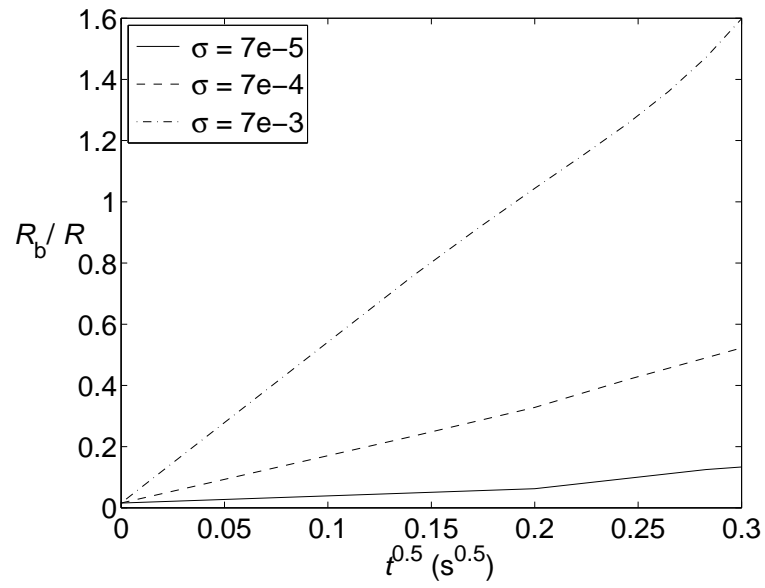

Fig. 4 The bridge radius versus the square root of time for the droplets coalescence problem with three different surface tension coefficients on a grid size of 64 for $R$.

To further access the effect of surface tension on the scaling law of the bridge radius, we use three different surface tension coefficients $\left(7 \times 10^{-5}\right.$, $7 \times 10^{-4}, 7 \times 10^{-3}$ ) on a grid size of 64 for $R$. As shown in Fig. 4, surface tension does not significantly influence the linear relationship of the bridge radius and the square root of time, but only influences the speed of coalescence. This agrees with the conclusion in Refs. 18, 20.

\section{Evolution of an initially circular fluid patch}

To verify LB-PLSM, the evolution of an initially circular fluid patch is investigated. This benchmark case is designed to examine the ability of a freesurface model to track the movement of the interface $^{21}$. An initially circular fluid patch is inviscid with a prescribed velocity field

$$
\begin{array}{r}
u_{0}(x, y)=-A_{0} x, \\
v_{0}(x, y)=A_{0} y,
\end{array}
$$

and a pressure field

$$
P_{0}=\frac{1}{2} \rho A_{0}^{2}\left[R^{2}-\left(x^{2}+y^{2}\right)\right] .
$$

As time goes on, the position of a fluid point at time $t$ is

$$
\mathbf{r}=\left(\begin{array}{l}
x(t) \\
y(t)
\end{array}\right)=\left(\begin{array}{c}
\frac{a(t)}{R} x_{0} \\
\frac{b(t)}{R} y_{0}
\end{array}\right),
$$

where $a(t)$ and $b(t)$ represent the semi-minor and semi-major axes of the elliptical fluid patch, and $x_{0}$ and $y_{0}$ are the initial coordinates of the fluid point. 
According to the mass conservation law, we can obtain the velocity field at time $t$ through some numerical operations:

$$
u(x, y, t)=-A(t) x, v(x, y, t)=A(t) y,
$$

where $A(0)=A_{0}$, and $A(t)$ is

$$
A(t)=-\frac{\mathrm{d} a}{\mathrm{~d} t} \frac{1}{a(t)}=\frac{\mathrm{d} b}{\mathrm{~d} t} \frac{1}{b(t)} .
$$

The pressure can be obtained by solving the pressure gradient from the Euler equation

$$
\begin{aligned}
P(x, y, t)= & \frac{1}{2} \rho\left[\frac{\mathrm{d} A}{\mathrm{~d} t}\left(x^{2}-y^{2}\right)-\right. \\
& \left.A^{2}\left(x^{2}+y^{2}\right)-a^{2}\left(\frac{\mathrm{d} A}{\mathrm{~d} t}-A^{2}\right)\right]
\end{aligned}
$$

with initial pressure $(\mathrm{d} A / \mathrm{d} t)(0)=0$.

Finally, imposing a null pressure all over the fluid patch, we can obtain a second-order nonlinear ordinary differential equation (ODE) for $A$ :

$$
\frac{\mathrm{d}^{2} A}{\mathrm{~d} t^{2}} A(t)-4\left(\frac{\mathrm{d} A}{\mathrm{~d} t}\right)^{2}+2 A^{4}(t)=0,
$$

initial conditions: $A(0)=A_{0}, \quad \frac{\mathrm{d} A}{\mathrm{~d} t}(0)=0$.

This ODE for $A$ can be solved by a fourth-order Runge-Kutta method. Since $A(t)$ is known, $a(t)$ and $b(t)$ can be obtained by solving

$$
a(t)=R \exp \left(-\int_{0}^{t} A(t) \mathrm{d} t\right)
$$

and

$$
b(t)=R \exp \left(\int_{0}^{t} A(t) \mathrm{d} t\right) .
$$

The simulation time is up to $t=2 / A_{0}$ with LB-PLSM. The radius of the circular droplet is $R=0.005 \mathrm{~m}$ and $A_{0}=5 \mathrm{~s}^{-1}$; the viscosity is $1 \times 10^{-6} \mathrm{~m}^{2} / \mathrm{s}$. To run the simulation, physical parameters are converted to dimensionless parameters. The simulations are implemented on a uniform grid with space step $\Delta x=1$ and time step $\Delta t=1$. Four different grid resolutions for $R(50,100,150,200)$ with corresponding dimensionless relaxation time coefficient $\tau(0.560,0.596,0.608,0.620)$ are adopted.

In Fig. 5 , at dimensionless time $t A_{0}=0$ and 0.5 , as to the position of the free-surface, the numerical result by LB-PLSM agrees well with the analytic solution. However, at $t A_{0}=1.0$ and 1.5 , the discrepancy between LB-PLSM and the analytical solution
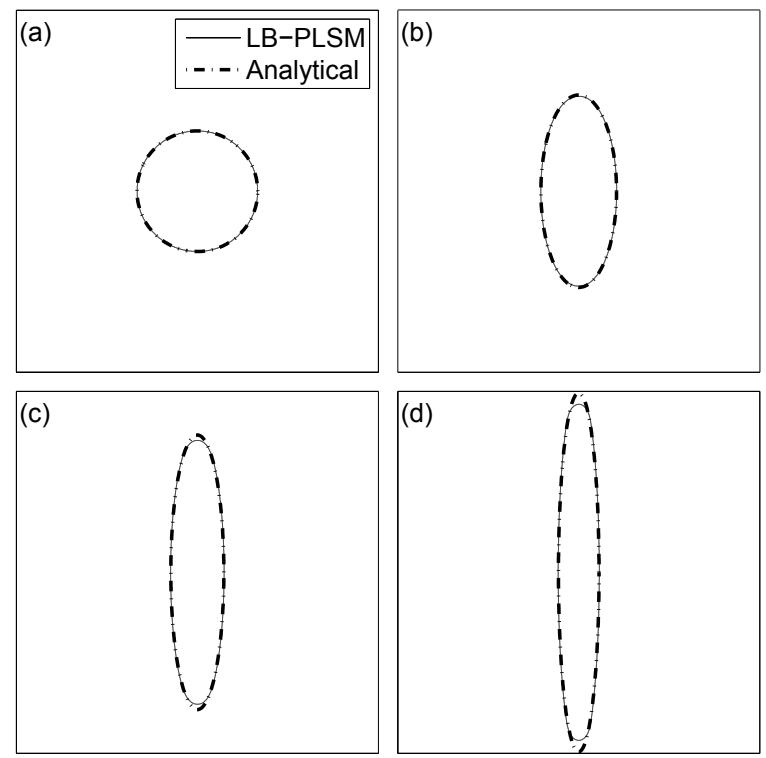

Fig. 5 Evolution of the initial circular fluid patch (case 4 with grid 200 for $R$ ) at dimensionless time (a) 0, (b) 0.5, (c) 1.0, (d) 1.5 .

becomes bigger. The cause for this discrepancy may be the viscosity which will slow down the evolution of the free-surface. Since the analytical solution is obtained with an inviscid condition, even with very low viscosity $\left(1 \times 10^{-6} \mathrm{~m}^{2} / \mathrm{s}\right.$ here $)$, the numerical result can still have a large difference with the analytical solution due to accumulation after a long simulation.

Fig. 6 displays the time history of the fluid velocity at points $(0.5 R, 0)$ and $(0,0.5 R)$ during the evolution of the fluid patch with four different grid resolutions. The numerical result by LB-PLSM is compared with the analytical solution. Although oscillating, vertical velocities for point $(0,0.5 R)$ and horizontal velocities for point $(0.5 R, 0)$ of all the four cases are in good agreement with the analytical solutions. In addition, with the increment of the grid resolution, the oscillating amplitudes decrease, which shows the convergence of the LB-PLSM model.

\section{Breaking dam}

The classical breaking dam case ${ }^{22,23}$ is simulated by LB-PLSM. To make a comparison, the lattice Boltzmann based single-phase free-surface model (LB-SP) proposed in Refs. 5,6 is also adopted to simulate this breaking dam case. The setup of the breaking dam case is similar to that in Janssen's work ${ }^{23}$. The physical model of the breaking dam is shown in Fig. 7 $(a=0.01 \mathrm{~m})$. Density of the water column is $1000 \mathrm{~kg} / \mathrm{m}^{3}$, viscosity is $1 \times 10^{-6} \mathrm{~m}^{2} / \mathrm{s}$, and gravity 

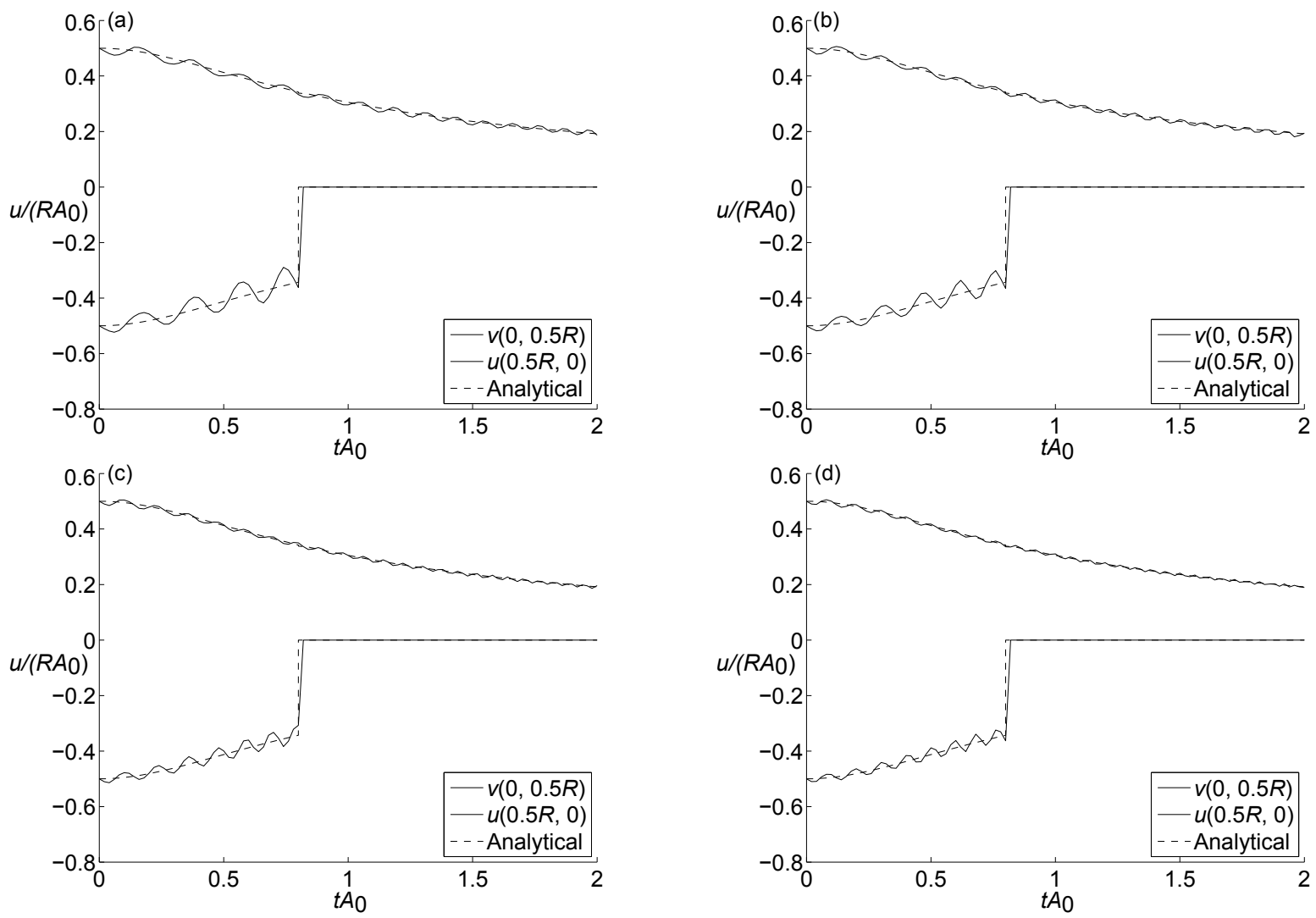

Fig. 6 Dimensionless velocity of the fluid patch at points $(0,0.5 R)$ and $(0.5 R, 0)$ from dimensionless time $0-2$ with grids (for circular radius $R$ ) of (a) 50, (b) 100, (c) 150, (d) 200. $v(0,0.5 R$ ) is in the higher part of this figure, while $u(0.5 R, 0)$ is in the lower part.

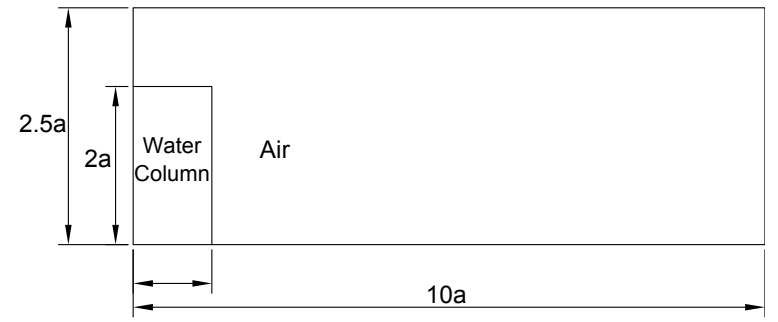

Fig. 7 The physical model of the breaking dam case $(a=$ $0.01 \mathrm{~m})$.

$g$ is $9.81 \mathrm{~m} / \mathrm{s}^{2}$. The dimensionless LB parameters are space step $(\Delta x=1)$ and time step $(\Delta t=1)$; three grid resolutions $(100 \times 25,200 \times 50,300 \times 75)$ with corresponding relaxation time coefficient $\tau(0.509$, $0.536,0.581)$; and gravity $\left(8.83 \times 10^{-5}, 1.77 \times 10^{-4}\right.$, $\left.2.65 \times 10^{-4}\right)$. Gravity is considered as a body force and is simulated by Guo's method (Eq. (6)-(8)) ${ }^{15}$. Slip boundary conditions are imposed on the front, bottom, and back walls. Simulations are stopped when water touches the front wall.
The evolutionary processes of the breaking dam (grid $300 \times 75$ ) of LB-PLSM and LB-SP are shown in Fig. 8. From Fig. 8, the free-surface simulated by LB-PLSM is much smoother than LB-SP. In Fig. 9 and Fig. 10, comparison between the experimental results from Ref. 22 and the numerical results of three different grid resolutions are made. The numerical results agree well with the experimental results, except at the surge front position with the $100 \times 25$ grid at the last few steps for LB-PLSM. This discrepancy results from the failure of capturing the high speed flow at the last few steps with a grid which is too small.

To further analyse the accuracy of LB-PLSM and LB-SP, we use two statistical parameters: amplitude $A_{F}$ and phase $P_{F}{ }^{24}$.

$$
\begin{gathered}
A_{F}=\sqrt{\sum_{i=1}^{N}\left(F_{i}^{\text {num }}\right)^{2} / \sum_{i=1}^{N}\left(F_{i}^{\exp }\right)^{2}} \\
P_{F}=\sqrt{\sum_{i=1}^{N}\left(F_{i}^{\text {num }}-F_{i}^{\text {exp }}\right)^{2} / \sum_{i=1}^{N}\left(F_{i}^{\exp }\right)^{2}}
\end{gathered}
$$



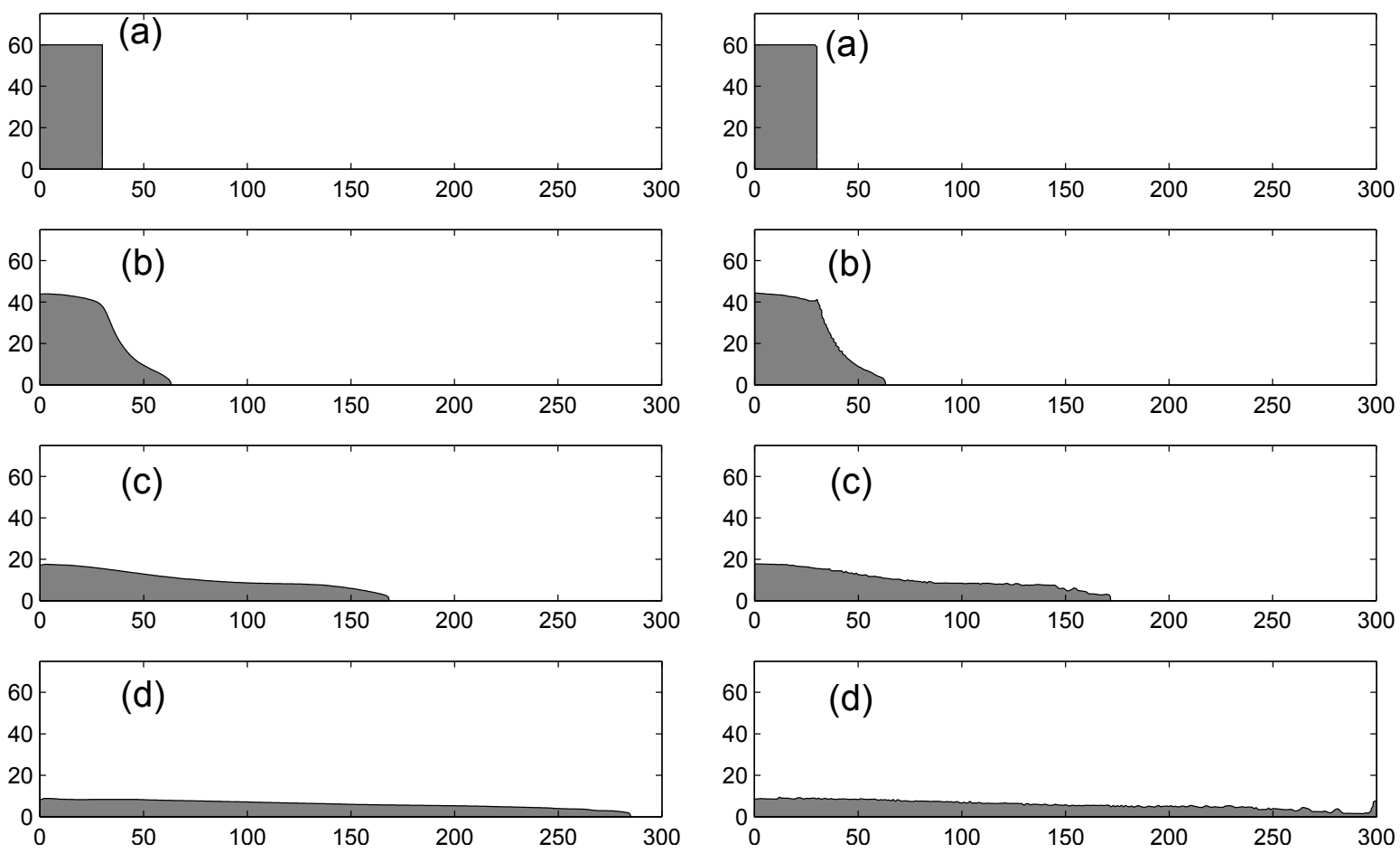

Fig. 8 The process of the breaking dam (grid: $300 \times 75$ ) at time step (a) 0 (b) 400 (c) 1000 (d) 1600. Left: LB-PLSM; right: LB-SP.
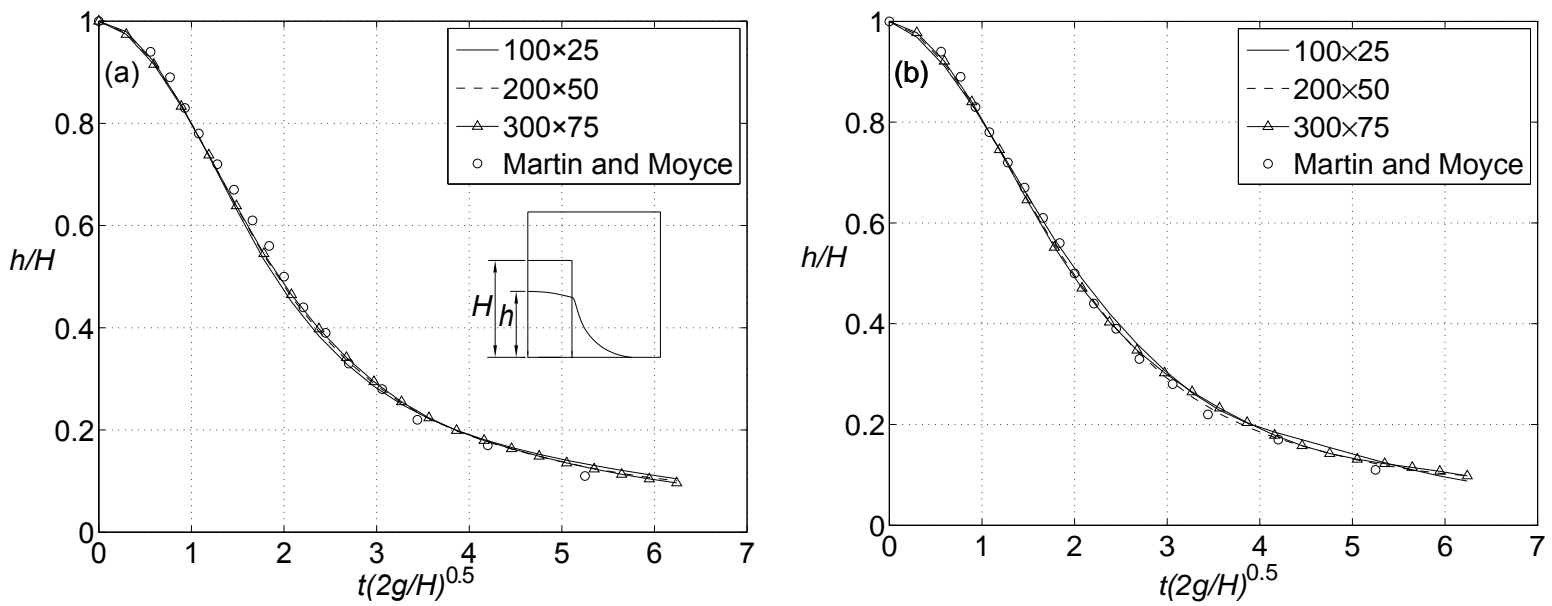

Fig. 9 Comparison of numerical and experimental results for the position of the water column top: (a) LB-PLSM, (b) LB-SP.

where $F_{i}$ is the magnitude to be examined (water top and surge front in this breaking dam case), $F_{i}^{\text {num }}$ are numerical results, $F_{i}^{\exp }$ are experimental results, and $N$ is the number of points to be analysed.

The values of these parameters for water top and surge front by LB-PLSM and LB-SP are displayed in Table 1. An exact agreement between numerical and experimental values requires $A_{F}=1$ and $P_{F}=0$. For LB-PLSM, from Table 1, all amplitude values are close to 1.0 and the finest grid resolution case (case
3) gives the best results, which shows the convergence of LB-PLSM with increasing grid resolution. Phase parameters of the surge front by LB-PLSM also show the convergence. As to LB-SP, the amplitude values of the water top shown in Table 1 are slightly better than LB-PLSM, but the phase values are worse. In general, results from both methods are very close to experimental results, which means that the accuracy of both methods is good. In addition, the amplitude values of the surge front from both methods are not 

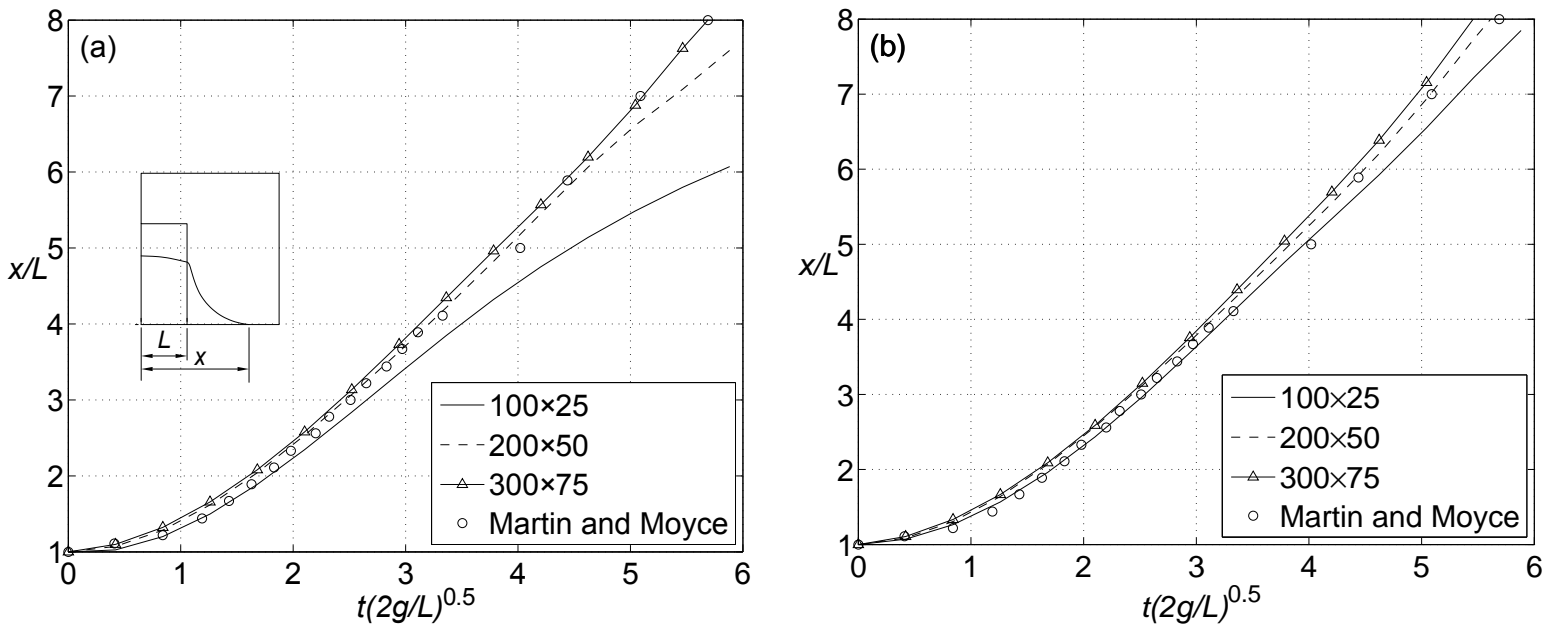

Fig. 10 Comparison of numerical and experimental results for the position of the surge front: (a) LB-PLSM, (b) LB-SP.

Table 1 Comparison of the water top and the surge front of the breaking dam case between experiment (exp) and calculation (num) by LB-PLSM and LB-SP.

\begin{tabular}{ccccccc}
\hline & \multirow{2}{*}{$\begin{array}{c}\text { Grid } \\
\text { resolution }\end{array}$} & \multicolumn{2}{c}{ Water top } & & \multicolumn{2}{c}{ Surge front } \\
\cline { 3 - 4 } & $A_{F}$ & $P_{F}$ & & $A_{F}$ & $P_{F}$ \\
\hline LB-PLSM & $100 \times 25$ & 0.977 & 0.032 & & 0.627 & 0.519 \\
& $200 \times 50$ & 0.981 & 0.026 & & 0.821 & 0.377 \\
& $300 \times 75$ & 0.982 & 0.024 & 0.873 & 0.199 \\
\hline LB-SP & $100 \times 25$ & 0.988 & 0.051 & 0.733 & 0.408 \\
& $200 \times 50$ & 0.990 & 0.042 & 0.856 & 0.203 \\
& $300 \times 75$ & 0.986 & 0.031 & 0.841 & 0.228 \\
\hline
\end{tabular}

as good as those of the water top, but these amplitude values are still close to 0.9 (at finest grid resolution). When the grid is $100 \times 25$, the amplitude values of the surge front from LB-PLSM are worse than LBSP. But with the increase of the grid resolution, the accuracy of LB-PLSM is better than that of LB-SP.

To test the efficiency of both methods, the total running time of each case for LB-PLSM and LBSP was noted. All the cases were implemented on the same PC (Intel Core i3-2100 CPU) without output operations. Each case was run six times and the final time for each case is the average of these six values. For different grid resolutions, $100 \times 25$, $200 \times 50,300 \times 75$, the total running time (in seconds) of LB-PLSM was 3, 9, 19 and LB-SP is 1.2, 4.3, 9.5. It is shown that LB-SP is nearly twice as fast as LBPLSM. Hence we could conclude that with enough grid resolution, LB-PLSM has a better accuracy but lower efficiency than LB-SP. If higher accuracy is required, LB-PLSM is a better choice.

\section{CONCLUSIONS}

In this paper, a coupled lattice Boltzmann and particle level set single-phase free-surface model has been developed and used to simulate the coalescence of two stationary droplets, the evolution of an initially circular fluid patch, and the classical breaking dam case. Coalescence of two stationary droplets shows that LB-PLSM can model surface tension for real freesurface problems. Evolution of an initially circular fluid patch suggests that the LB-PLSM can track the free-surface accurately. As to the breaking dam case, numerical results by LB-PLSM are compared with experimental results and the results by LB-SP, which shows that LB-PLSM has better accuracy but lower efficiency than LB-SP. However, to get more stable and accurate results, the large eddy model (LES) and multiple relaxation time model can be used. Furthermore, to improve the efficiency of LB-PLSM, parallelism of CPU and GPU can be implemented. These improvements and the extension of LB-PLSM to three dimensions will be presented elsewhere.

Acknowledgements: We acknowledge the financial support from the National Natural Science Foundation of China (grant no. 50975103), the National Natural Science Funds for Distinguished Young Scholars (grant no. 51006039) and the generous support from Huazhong University of Science and Technology.

\section{REFERENCES}

1. Gunstensen AK, Rothman DH, Zaleski S, Zanetti G (1991) Lattice Boltzmann model of immiscible fluids. Phys Rev A 43, 4320-7. 
2. Shan X, Chen H (1993) Lattice Boltzmann model for simulating flows with multiple phases and components. Phys Rev E 47, 1815-9.

3. Swift MR, Osborn WR, Yeomans JM (1995) Lattice Boltzmann simulation of nonideal fluids. Phys Rev Lett 75, 830-3.

4. Ginzburg I, Steiner K (2003) Lattice Boltzmann model for free-surface flow and its application to filling process in casting. J Comput Phys 185, 61-99.

5. Körner C, Thies M, Hofmann T, Thürey N, Rüde U (2005) Lattice Boltzmann model for free surface flow for modeling foaming. J Stat Phys 121, 179-96.

6. Thürey N, Rüde U, Körner C (2005) Interactive free surface fluids with the lattice Boltzmann method. Technical Report 05-4, Univ of Erlangen-Nuremberg, Germany.

7. Osher S, Sethian JA (1988) Fronts propagating with curvature-dependent speed: algorithms based on Hamilton-Jacobi formulations. J Comput Phys 79, 12-49.

8. Sethian JA (1996) Level Set Methods and Fast Marching Methods, Cambridge Univ Press, Cambridge.

9. Adalsteinsson D, Sethian JA (1999) The fast construction of extension velocities in level set methods. J Comput Phys 148, 2-22.

10. Sussman M, Puckett EG (2000) A coupled level set and volume-of-fluid method for computing 3D and axisymmetric incompressible two-phase flows. J Comput Phys 162, 301-37.

11. Enright D, Fedkiw R, Ferziger J, Mitchell I (2002) A hybrid particle level set method for improved interface capturing. J Comput Phys 183, 83-116.

12. Enright D, Losasso F, Fedkiw R (2005) A fast and accurate semi-Lagrangian particle level set method. Comput Struct 83, 479-90.

13. Bhatnagar PL, Gross EP, Krook M (1954) A model for collision processes in gases. I. Small amplitude processes in charged and neutral one-component systems. Phys Rev 94, 511-25.

14. Qian YH, D'Humieres D, Lallemand P (1992) Lattice BGK models for Navier-Stokes equation. Europhys Lett 17, 479-84.

15. Guo Z, Zheng C, Shi B (2002) Discrete lattice effects on the forcing term in the lattice Boltzmann method. Phys Rev E 65, 046308.

16. Thürey N, Rüde U (2004) Free surface lattice-Boltzmann fluid simulations with and without level sets. In: Girod B, Magnor M, Seidel H-P (eds) Vision Modeling and Visualization 2004 (VMV 2004 Proceedings, Standford, USA), IOS Press, pp 199-208.

17. Kwak Y, Kuo CCJ, Nakano A (2009) Hybrid latticeBoltzmann/level-set method for liquid simulation and visualization. Int J Comput Sci 3, 579-92.

18. Eggers J, Lister JR, Stone HA (1999) Coalescence of liquid drops. J Fluid Mech 401, 293-310.

19. Wu M, Cubaud T, Ho CM (2004) Scaling law in liquid drop coalescence driven by surface tension. Phys Fluids 16, L51-4.
20. Xing XQ, Butler DL, Ng SH, Wang Z, Danyluk S, Yang C (2007) Simulation of droplet formation and coalescence using lattice Boltzmann-based single-phase model. J Colloid Interface Sci 311, 609-18.

21. Colagrossi A (2004) A meshless Lagrangian method for free-surface and interface flows with fragmentation. $\mathrm{PhD}$ thesis, Universita di Roma, Rome.

22. Martin JC, Moyce WJ (1952) An experimental study of the collapse of liquid columns on a rigid horizontal plane. Phil Trans Roy Soc Lond Ser A 244, 312-24.

23. Janssen C, Krafczyk M (2010) A lattice Boltzmann approach for free-surface-flow simulations on nonuniform block-structured grids. Comput Math Appl 59, 2215-35.

24. Crespo AC, Dominguez JM, Barreiro A, GómezGesteira M, Rogers BD (2011) GPUs, a new tool of acceleration in CFD: Efficiency and reliability on smoothed particle hydrodynamics methods. PLOS ONE 6, e20685. 